\title{
SUPPRESSION OF 12-O-TETRADECANOYLPHORBOL-13-ACETATE INDUCED ORNITHINE DECARBOXYLASE ACTIVITY BY RESVERATROL DERIVATIVES
}

Suaib Luqman ${ }^{1,2}$, Tamara P. Kondratyuk¹, Juma Hoshino³, Mark Cushman³, John M. Pezzuto*

College of Pharmacy, University of Hawaii at Hilo, Hilo, Hawaii 96720, United States.

Molecular Bioprospection Department of Biotechnology Division, CSIR-Central Institute of Medicinal and Aromatic Plants, Lucknow-226015, India.

Department of Medicinal Chemistry and Molecular Pharmacology, College of Pharmacy, and The Purdue University Center for Cancer Research, Purdue University, West Lafayette, Indiana 47907, United States.

\section{INTRODUCTION}

Resveratrol (3,4,5-trihydroxy-trans-stilbene), a phytoalexin found in grape skins, peanuts, and red wine, have a potent chemopreventive effect in multiple carcinogenesis models .

Resveratrol and its analogues are known to interfere with signal transduction pathways, where they inhibit activities of various protein kinases which in turn declines the expression of nuclear proto-oncogenes and the activity of ornithine decarboxylase (ODC) is reduced.

ODC activity and expression have been among the first biomarkers of neoplastic proliferation and catlyzes the rate- limiting step in polyamine biosynthesis.

DC activity is essential for cell proliferation and is required for progression into the $S$ phase of the cell cycle.

PAA induction of ODC mRNA expression and ODC activity may be PKC activation dependent or independent.

\section{RESULTS}

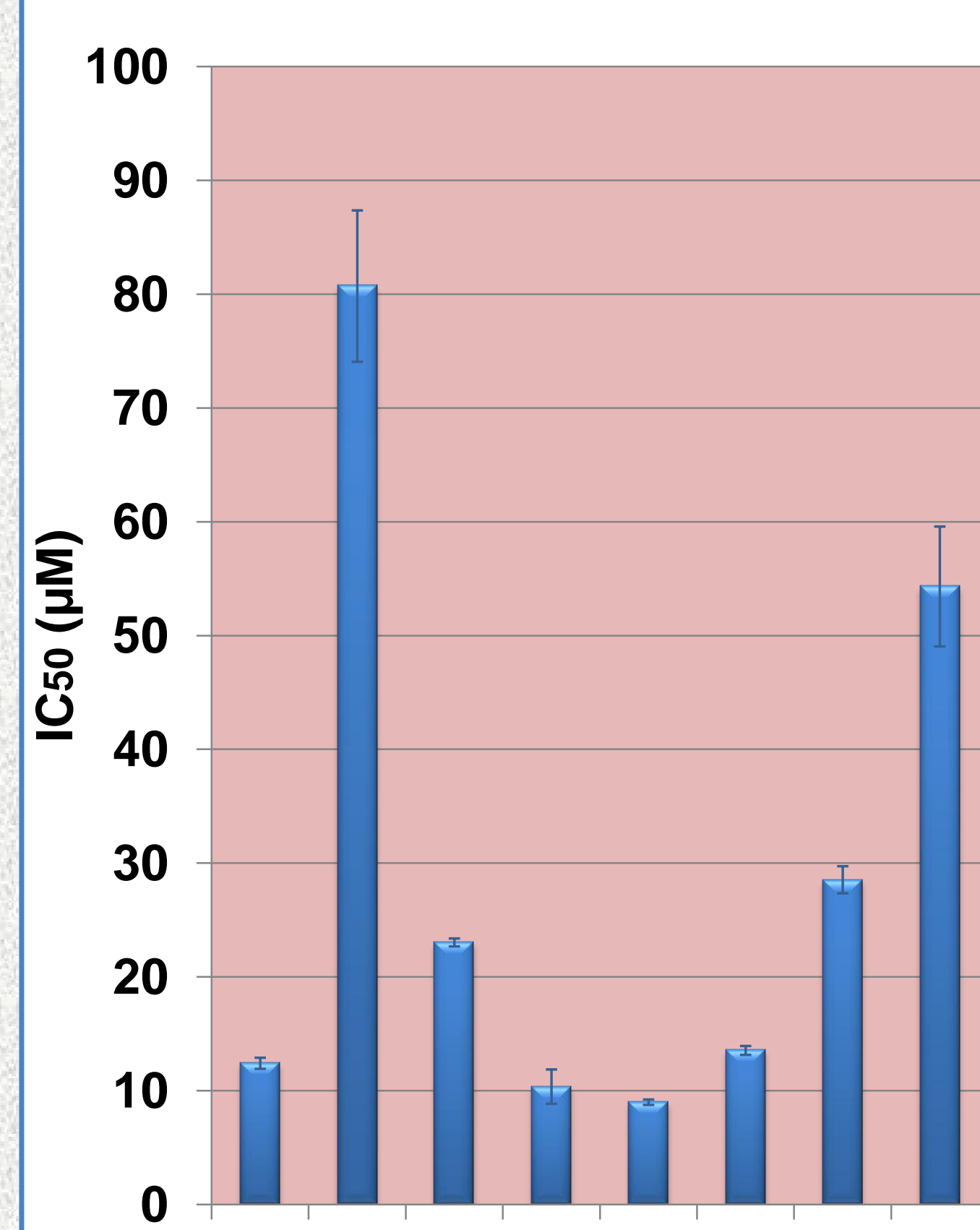

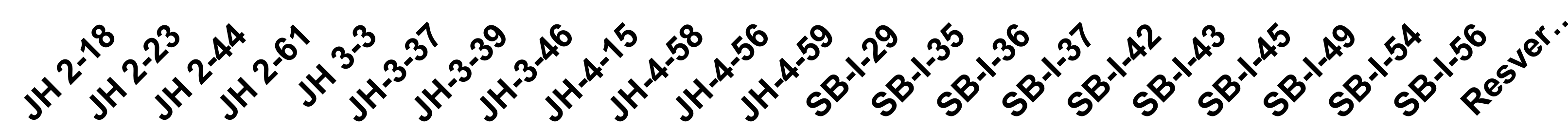

\begin{tabular}{|c|c|c|c|}
\hline Compounds & Inhibition (\%) & Surviv & val (\%) \\
\hline JH 2-18 & $94.27 \quad 0.55$ & 68.2 & 14.3 \\
\hline JH 2-23 & $90.55 \quad 4.49$ & 71.9 & 17.2 \\
\hline JH 2-44 & $\begin{array}{ll}98.15 & 0.08\end{array}$ & 99.5 & 4.1 \\
\hline JH 2-61 & $\begin{array}{ll}97.85 & 0.66\end{array}$ & 56.6 & 16.7 \\
\hline JH 3-3 & $96.17 \quad 1.3$ & 60.5 & 19.1 \\
\hline JH-3-37 & $\begin{array}{ll}95.07 & 0.97\end{array}$ & 67.0 & 18.6 \\
\hline JH-3-39 & $90.8 \quad 2.15$ & 66.4 & 22.9 \\
\hline JH-3-46 & $96.2 \quad 0.44$ & 78.1 & 11.4 \\
\hline JH-4-15 & $\begin{array}{ll}95.12 & 0.41\end{array}$ & 74.4 & 8.7 \\
\hline JH-4-58 & $93.52 \quad 0.14$ & 73.8 & 3.1 \\
\hline JH-4-56 & $\begin{array}{ll}97.27 & 0.03\end{array}$ & 84.4 & 17.5 \\
\hline JH-4-59 & $91.07 \quad 2.7$ & 89.6 & 7.1 \\
\hline SB-I-29 & $90.34 \quad 6.5$ & 48.1 & 12.0 \\
\hline SB-I-35 & $\begin{array}{ll}95.42 & 0.23\end{array}$ & 58.0 & 2.6 \\
\hline SB-I-36 & $\begin{array}{ll}95.35 & 1.15\end{array}$ & 64.1 & 8.0 \\
\hline SB-I-37 & $\begin{array}{ll}95.80 & 0.58\end{array}$ & 79.8 & 5.2 \\
\hline SB-I-42 & $94.87 \quad 2.39$ & 52.9 & 9.2 \\
\hline SB-I-43 & $\begin{array}{ll}92.26 & 0.34\end{array}$ & 58.7 & 25.0 \\
\hline SB-I-45 & $93.49 \quad 3.92$ & 77.7 & 6.3 \\
\hline SB-I-49 & $\begin{array}{ll}97.86 & 0.45\end{array}$ & 94.9 & 30.2 \\
\hline SB-I-54 & $\begin{array}{ll}47.62 & 7.2 \\
\end{array}$ & 53.4 & 10.5 \\
\hline SB-I-56 & $\begin{array}{ll}97.78 & 0.24\end{array}$ & 62.6 & 14.9 \\
\hline Resveratrol & $96.09 \quad 1.82$ & 99.1 & 11.7 \\
\hline
\end{tabular}

Inhibition of TPA induced Ornithine decarboxylase activity

* JH 4-15 showed maximum inhibition of ODC activity at IC50 $1.21 \mu \mathrm{M}$ following order: $J H 2-23>J H 3-46>S B|49>S B| 43>S B I 37>S B I 56>$ SB I6>JH 3-39>JH 2-44>SB I 42>SB I 45>SB I54>JH 3-37>JH 2-

18>JH 2-61>JH 3-3>SB I 29>SB I 35>JH 4-58>JH 4-56> JH 4-59>JH 4-

\section{CONCLUSION}

* Our studies depicts potential metabolites having greater activity [tetrabutylammonium (E)-4-(3,5-dihydroxystyryl)phenyl sulfate (IC50 $1.2 \mu \mathrm{MM})$, resveratro tripotassium 3,5,4'-trisulfate (IC50 $1.8 \mu \mathrm{M}$ ), resveratrol tripotassium 3,4'-disulfate (IC50 $1.8 \mu \mathrm{M})$, and resveratrol tripotassium 3,5-disulfate (IC50 $2.3 \mu \mathrm{M})]$, than the

- TPA-increased amount of ODC mRNA may be the result of enhanced ODC gene transcription and/or decreased degradation of ODC mRNA.

During ODC induction by TPA, the increase in its MRNA is usually much less than the observed activity, suggesting that some regulation of ODC activity occurs posttranscriptionally.

Some of the resveratrol derivatives, doesnot show any significant
$1.2 \mu \mathrm{M}$, suggesting their mode of action which is PKC independent.
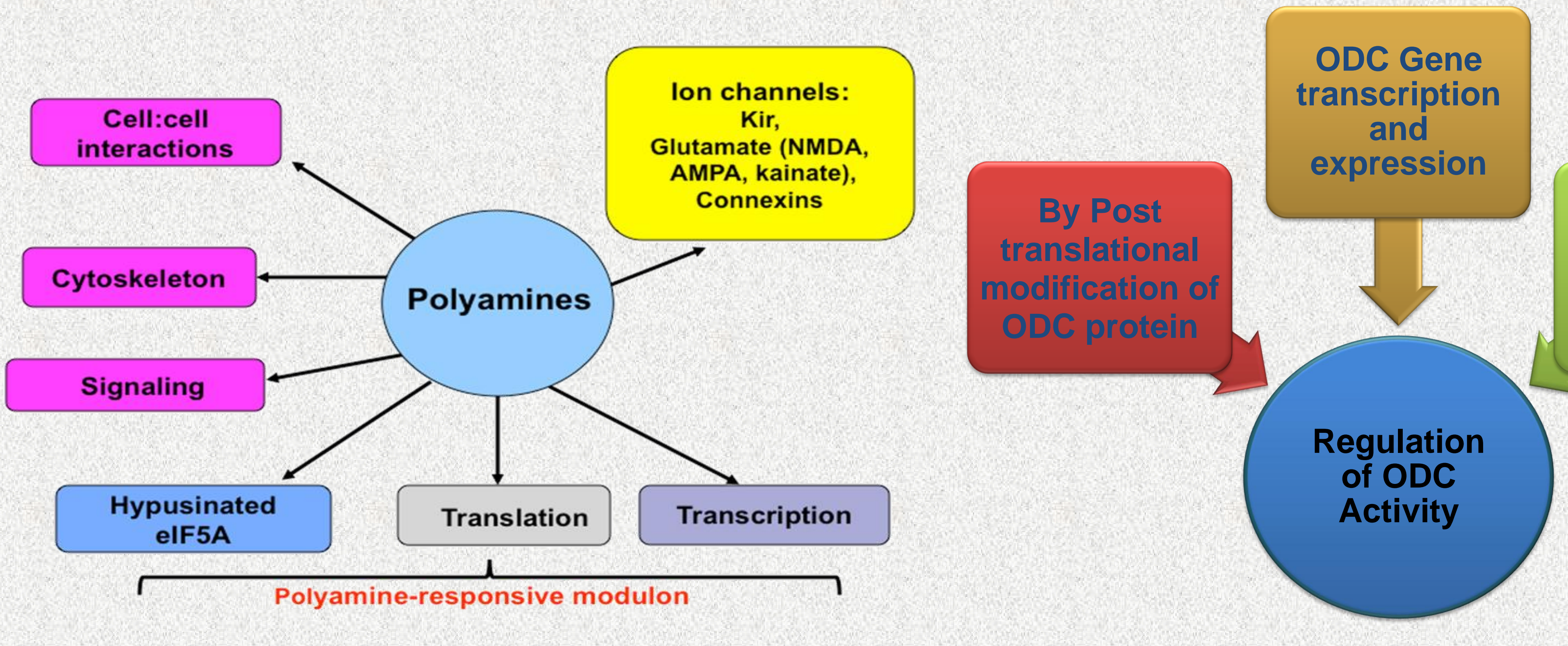

EXPERIMENTAL REFERENCES

Nature Medicine 1995, 1: 260-266.

Cancer Research 1997, 57: 272-278.

Journal of Biochemistry 2002, 132: 663-668.

Annals of New York Academy of Sciences 2003, 987:

125-134.
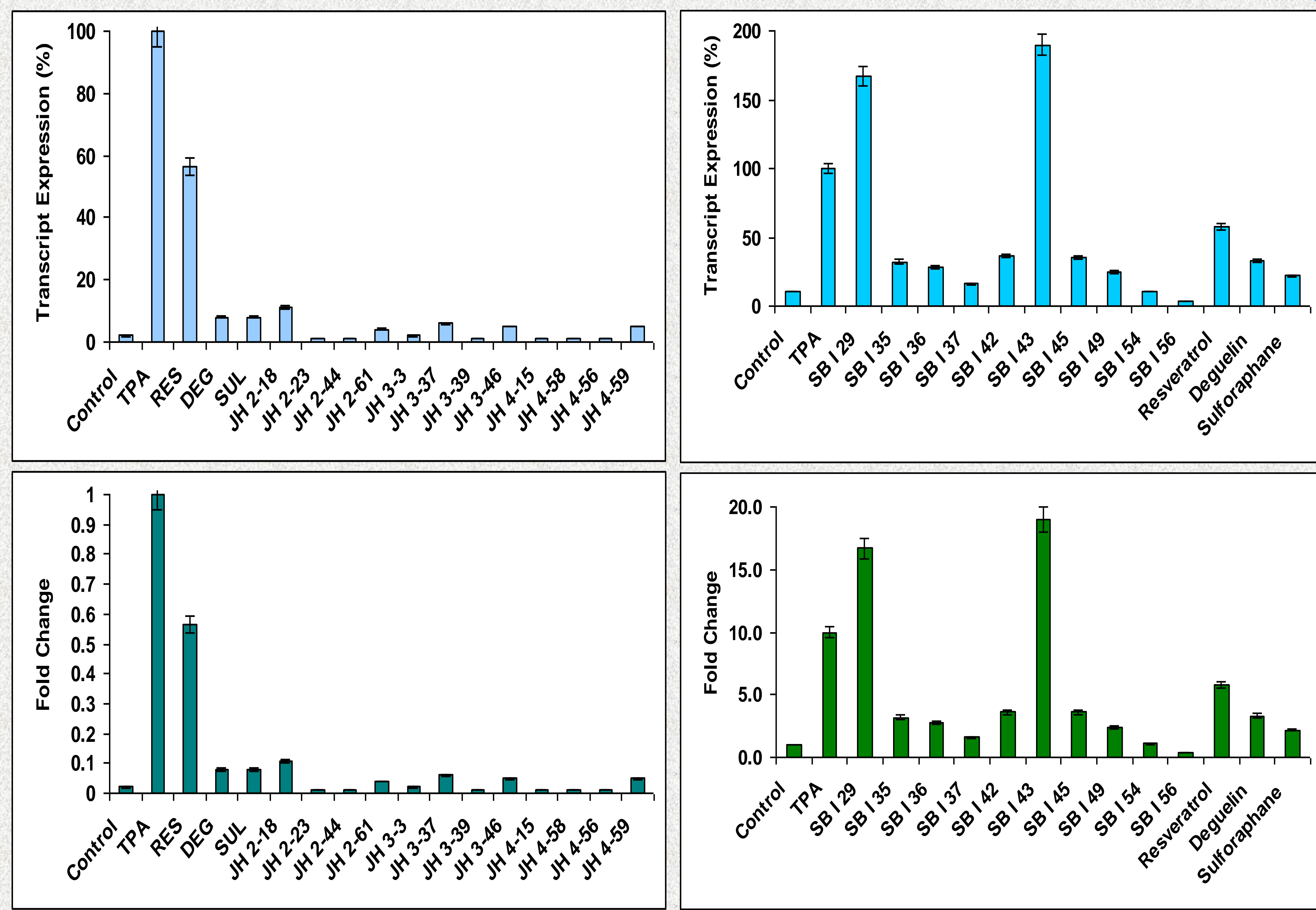

RRA Expression Analysis

$\mathrm{JH} 2-23$ showed highest inhibition $(99.0 \pm 5.0 \%)$ and Resveratrol lowest $(58.0 \pm 2.6$ $\%$.

2-61> JH 4-59> JH 3-46> JH 3-37> SB I-54> JH 2-18> SB I-37> SB I-49> SB I-36> SB I-35> SB I-45> SB
(

(

HILO

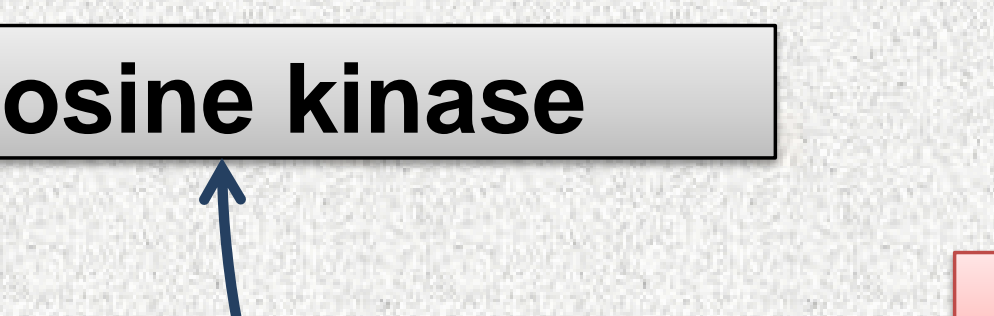

Spermine

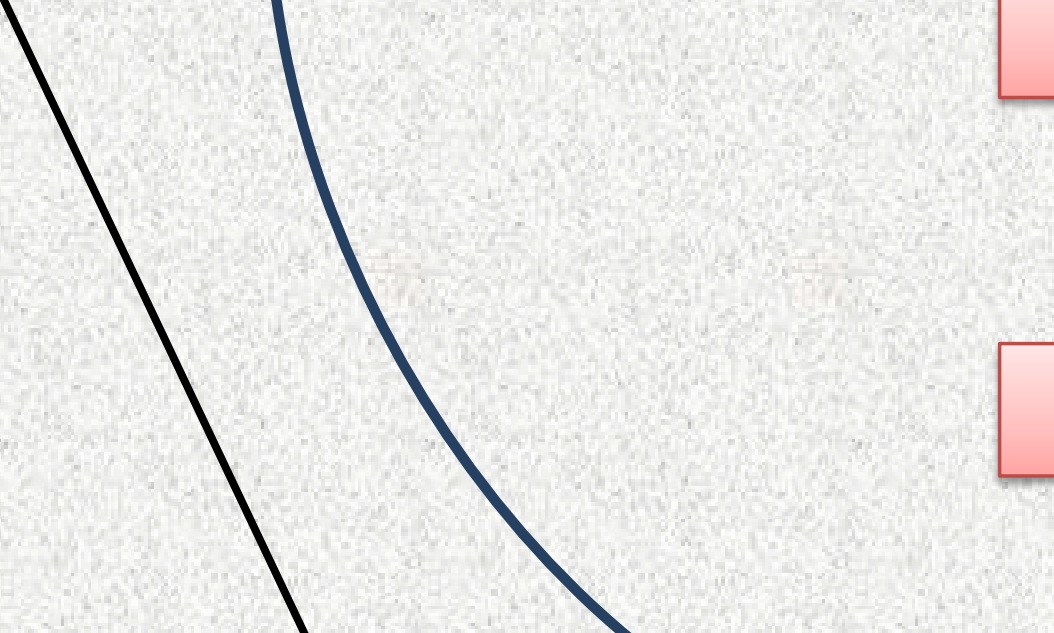

Putrescine ODC

Ornithine 